\title{
Acute Lyme Arthritis Presenting in Bilateral Hips of an Elderly Patient: A Case Report
}

\author{
Lawrence M Asprec ${ }^{1 *}$, Mitchell K Freedman ${ }^{2}$, Jeffrey A Gehret ${ }^{3}$ and George W Young ${ }^{3}$
}

${ }^{1}$ Senior Resident in Physical Medicine and Rehabilitation, Thomas Jefferson University Hospital at Sidney Kimmel Medical College, Philadelphia, USA

${ }^{2}$ Medical Director of Physical Medicine and Rehabilitation, Associate Professor, Rothman Orthopaedic Institute, 925 Chestnut Street, 5th Floor, Philadelphia, USA

${ }^{3}$ Department of Physical Medicine and Rehabilitation, Division of Pain Management, Rothman Orthopaedic Institute, 925 Chestnut Street, 5th Floor, Philadelphia, USA

*Corresponding author: Lawrence M. Asprec, MD, Resident Physician, Department of Rehabilitation Medicine Thomas Jefferson University Hospital, Sidney Kimmel Medical College, 25 S. Ninth Street, Philadelphia, PA, 19107, USA, Tel: 215955-6585; 908-642-4754, Fax: 215-955-2311

\section{Abstract}

Introduction: We describe a case report of an elderly patient who had presented with acute onset of atypical lower extremity pain and gait dysfunction, and was ultimately diagnosed with Lyme arthritis.

Case report: A 70-year-old male presented with a chief complaint of 10 days of lower back and bilateral lower extremity pain. He described sudden onset of intense, sharp bilateral anterior thigh pain radiating to the medial calves intermittently and without paresthesia, bowel or bladder incontinence, fevers, rashes or weight change. His gait was found to be antalgic and slow. Strength, sensation, and reflexes were intact in both legs. X-ray imaging of bilateral hips revealed mild osteoarthritis bilaterally. An MRI of the bilateral hips showed bilateral hip effusions with synovial proliferation and degenerative labral tearing. He underwent a fluoroscopically guided bilateral injection with each hip, and clear, straw-colored synovial fluid was aspirated from the left hip. He gave additional history following the injection of being treated for Lyme disease 4 months prior to the onset of his leg pain, for which he previously completed 2 weeks of doxycycline. Fluid analysis revealed elevated nucleated cell count and positive serology for multiple Borrelia-specific bands, consistent with Lyme arthritis. He was subsequently treated with a 4 week course of doxycycline. As of 18 months later, his pain has improved significantly.

Conclusion: Patients with antibiotic refractory Lyme disease that continue to have chronic symptoms, including arthritis and synovitis, after appropriate antibiotic therapy may benefit from treatment with anti-inflammatory medications, disease-modifying anti-rheumatic drugs (DMARD) or synovectomy.
\end{abstract}

\section{Keywords \\ Lyme arthritis}

\section{Introduction}

Lyme disease is the most common tick-borne illness of the spirochete Borrelia burgdorferi in the United States and Europe, and most often cultivated in the late spring and early summer seasons [1]. Clinical manifestations of localized disease include headache and fatigue in addition to the classic skin lesion, erythema migrans early in the disease [1]. Disseminated infection evolves into neurologic and cardiac findings. Later stages of the disease, specifically Lyme arthritis, are characterized by brief, recurrent episodes of unilateral or asymmetric oligoarthritis, most commonly affecting the knee, and more commonly found in children than adults $[2,3]$. We describe a case report of an elderly patient who had presented with acute onset of atypical lower extremity pain and gait dysfunction, and was ultimately diagnosed with Lyme arthritis.

\section{Case Report}

A 70-year-old male with past medical history of diabetes mellitus type II, hypertension, and hyperlipidemia presented to our outpatient center with a chief

Citation: Asprec LM, Freedman MK, Gehret JA, Young GW (2019) Acute Lyme Arthritis Presenting in Bilateral Hips of an Elderly Patient: A Case Report. Int J Physiatry 5:018. doi.org/10.23937/25724215.1510018

Accepted: December 07, 2019: Published: December 09, 2019

Copyright: (c) 2019 Asprec LM, et al. This is an open-access article distributed under the terms of the Creative Commons Attribution License, which permits unrestricted use, distribution, and reproduction in any medium, provided the original author and source are credited. 
complaint of 10 days of lower back and bilateral lower extremity pain. He described sudden onset of intense, sharp bilateral anterior thigh pain radiating to the medial calves intermittently. He denied paresthesia. The pain was worse at night and when changing positions from sitting to standing, but improved with walking. He denied bowel or bladder incontinence, fevers, rashes or weight change. His primary care physician initiated a course of methylprednisolone and atorvastin was discontinued, resulting in mild improvement in pain. On physical examination, he was found to have pain with range of motion of the hips, left greater than right. He did not have any focal tenderness in the back or hips. His gait was found to be antalgic and slow. Strength, sensation, and reflexes were intact in both legs.

X-ray imaging of bilateral hips revealed mild osteoarthritis bilaterally. MRI of his lumbar spine was unchanged from a previous MRI and revealed multilevel spondylotic changes and grade I spondylolisthesis at L5-S1 in addition to a left foraminal disc herniation at the same level. An MRI of the bilateral hips showed bilateral hip effusions with synovial proliferation and degenerative labral tearing. He underwent a fluoroscopically guided bilateral hip injection with each hip receiving $40 \mathrm{mg}$ depo-medrol and $2 \mathrm{~mL}$ of $1 \%$ lidocaine; $6 \mathrm{~mL}$ of clear, straw-colored synovial fluid was aspirated from the left hip. He gave additional history following the injection of being treated for Lyme disease 4 months prior to the onset of his leg pain. He was treated with 2 weeks of doxycycline after the diagnosis based on a bullseye rash and positive serological testing. The fluid was sent for analysis and revealed elevated nucleated cell count of 15677, predominantly lymphocytes, and positive serology for a total of eight Borrelia-specific bands, consistent with Lyme arthritis. Synovial fluid crystals, cultures, and gram staining were negative. He was subsequently treated with a 4 week course of doxycycline. As of 18 months later, he has $2 / 10$ pain in the groin and anterior thigh bilaterally when changing positions from seated to standing.

\section{Discussion}

Hip pain is a common diagnosis in adults. The differential diagnoses of acute hip pain in the elderly include mechanical injury, osteoarthritis, avascular necrosis, transient synovitis, inflammatory arthritis, septic arthritis, gout, pseudogout and less likely the various seronegative spondyloarthropathies [1]. In certain endemic areas, specifically the Northeastern area of the United States and various areas of Europe (Slovenia, Austria, and Lithuania), Lyme disease is important to include in the differential [1].

Lyme arthritis is characterized by intermittent or persistent monoarthritis or asymmetric oligoarthritis frequently involving the knee, and less commonly the shoulders, ankles, elbow, temporomandibular joint, wrist, and hips [2]. Onset of arthritis, after developing erythema migrans, ranges from a few days to years with most cases occurring within the first three to six months $[2,4]$. Fewer than 5 joints are usually affected at one time with children having more joints affected than adults [5]. Knee swelling, warmth and an effusion in Lyme patients are typically not painful with range of motion on exam or weight bearing which helps differentiate it from septic arthritis [6]. Systemic symptoms, such as fever, headache and myalgias, are uncommon with generalized fatigue being the most common associated symptom [6]. Chronic joint dysfunction (cartilage or bone erosion) is rare even without treatment. However, up to $60 \%$ of patients will develop arthritis in the late stages of the disease if not previously treated [2].

Serologic testing is the mainstay in Lyme disease diagnosis [6-8]. The CDC recommends a two-step process where samples are first tested using enzyme-linked immunosorbent assay for antibodies to B burgdorferi. Samples with a positive or equivocal result are then tested by Western blotting for presence of IgM or IgG antibody responses with findings interpreted per CDC guidelines [7]. Due to Lyme arthritis being a late disease manifestation, patients will have IgG antibody responses. Synovial fluid analysis is achieved using polymerase chain reaction (PCR) testing for B. burgdorferi DNA, due to joint fluid cultures commonly resulting as negative. PCR testing of synovial fluid in Lyme arthritis patients are typically positive before antibiotic therapy and usually negative after appropriate antibiotic treatment [8]. Synovial fluid analysis is usually performed to rule out other entities such as gout and infection. Typical range for joint fluid white cell counts in Lyme arthritis is 10,000 to 25,000 cells $/ \mathrm{mm}^{3}$ [2]. Serologic testing, such as complete blood count and rheumatological factors, are usually negative with the possible exception of elevated inflammatory markers. Imaging studies are not necessary for Lyme arthritis diagnosis but can be performed to investigate for alternative diagnosis. In adult patients, $\mathrm{X}$-ray may show degenerative changes, but symptom severity is often out of proportion to arthritic severity; MRI may show synovial thickening and joint effusion [9]. For those patients that suffer with chronic Lyme arthritis, inflammatory joint destruction may be seen with periarticular osteoporosis, cartilage destruction and bone erosions [9].

Treatment of adults with Lyme arthritis is usually successful with a 28 day course of doxycycline $(100 \mathrm{mg}$ twice daily), amoxicillin (500 mg three times daily) or cefuroxime axetil (500 $\mathrm{mg}$ twice daily). An additional 4 weeks of oral antibiotics can be given to those patients whose symptoms have improved but are not completely resolved with 2-4 weeks of intravenous ceftriaxone reserved for those whose symptoms are unchanged or worsened. If an individual remains symptomatic after 
2 months of therapy, repeat PCR of joint fluid/tissue is recommended. When the PCR is negative, symptomatic treatment is recommended with use of NSAIDs, corticosteroid joint injection or disease-modifying anti-rheumatic drugs after consultation with a rheumatologist. Synovectomy is reserved for refractory cases of severe synovitis [10].

The above case is unique in a variety of ways. Lyme arthritis presenting in the hip has been primarily described in case reports in the pediatric population. Our case also demonstrates bilateral hip involvement with joint effusions which also has not been described. Children commonly have multiple joints involved at the time of diagnosis of Lyme arthritis, while adults often only have a single joint involved [6]. Furthermore, since the referring diagnosis was lumbar radiculopathy, the importance of an accurate history and physical must be emphasized. Lastly, in patients who present with joint pain or swelling following the treatment of Lyme disease, Lyme arthritis must be considered as a potential etiology. Despite the evolution of early detection and treatment of Lyme disease, associated arthritis is still the second most frequent manifestation of the disease [3].

Intra-articular injection of corticosteroid is usually reserved until after antibiotics have successfully eradicated the spirochete from the joint [6]. The additional history in this case that the patient was recently treated for acute Lyme disease was not discovered until after the injections were performed.

Fortunately, joint fluid was aspirated and analyzed with subsequent treatment with antibiotics and successful improvement in pain and function. However, this patient may be classified as antibiotic refractory Lyme arthritis and could potentially develop symptoms again, despite multiple courses of antibiotics [2].

Four weeks of oral antibiotics is first-line treatment of Lyme arthritis with refractory cases requiring an addition 4 weeks of oral antibiotic or 2-4 weeks of intravenous antibiotics [6]. For the few patients that continue to have chronic symptoms after appropriate antibiotic therapy, treatment with anti-inflammatory medications, disease-modifying anti-rheumatic drugs (DMARD) or synovectomy may be considered.

\section{Conclusion}

Patients with antibiotic refractory Lyme disease that continue to have chronic symptoms, including arthritis and synovitis, after appropriate antibiotic therapy may benefit from treatment with anti-inflammatory medications, disease-modifying anti-rheumatic drugs (DMARD) or synovectomy.

\section{Clinical Message}

In patients who present with joint pain or swelling following the treatment of Lyme disease, Lyme arthritis must be considered as a potential etiology. Despite the evolution of early detection and treatment of Lyme disease, associated arthritis is still the second most frequent manifestation of the disease.

\section{Statement of Informed Consent}

The patient was informed that data concerning the case would be submitted for publication and they agreed and consented.

\section{Competing Interests}

The authors declare that they have no competing interests.

\section{References}

1. Steere AC, Malawista SE, Snydman DR, Shope RE, Andiman WA, et al. (1977) Lyme arthritis: an epidemic of oigoarticular arthritis in children and adults in three connecticut communities. Arthritis Rheum 20: 7-17.

2. Steere AC, Schoen RT, Taylor E (1987) The clinical evolution of Lyme arthritis. Ann Intern Med 107: 725-731.

3. Bacon RM, Kugeler KJ, Mead PS (2008) Surveillance for Lyme disease-United States, 1992-2006. MMWR SurveillSumm 57: 1-9.

4. Feder HM Jr, Johnson BJ, O'Connell S, Shapiro ED, Steere AC, et al. (2007) A critical appraisal of chronic Lyme disease. N Engl J Med 357: 1422-1430.

5. Dart AH, Michelson KA, Aronson PL, Garro AC, Lee TJ, et al. (2018) LE Hip Synovial Fluid Cell Counts in Children From a Lyme Disease Endemic Area. Pediatrics 141: e20173810.

6. Daikh BE, Emerson FE, Smith RP, Lucase FL, McCarthy CA (2013) Lyme arthritis: a comparison of presentation, synovial fluid analysis, and treatment course in children and adults. Arthritis Care Res (Hoboken) 65: 1986-1990.

7. Centers for Disease Control and Prevention (1995) Recommendations for test performanceand interpretation from the Second International Conference on SerologicDiagnosis of Lyme Disease. MMWR Morb Mortal Wkly Rep 44: 590-591.

8. Nocton JJ, Dressler F, Rutledge BJ (1994) Detection of BorreliaburgdorferiDNAby polymerase chain reaction in synovial fluid in Lyme arthritis. N Engl J Med 330: 229-234.

9. Lawson JP, Steere AC (1985) Lyme arthritis: radiologic findings. Radiology 154: 37-43.

10. Wormser GP, Dattwyler RJ, Shapiro ED (2006) The clinical assessment, treatment, and prevention of Lyme disease, human granulocytic anaplasmosis, and babesiosis: clinical practice guidelines by the infectious diseases society of america. Clin Infect Dis 43: 1089-1134. 